\title{
Regulation of MUC5AC mucin production by the cell attachment dependent pathway involving integrin $\beta 1$ in NCI-H292 human lung epithelial cells
}

\author{
Jun Iwashita*, Kaori Hongo, Yuho Ito, Tatsuya Abe, Jun Murata
}

Faculty of Bioresource Sciences, Akita Prefectural University, Akita, Japan

Email: ${ }^{*}$ jun_iwashita@akita-pu.ac.jp

Received 9 November 2012; revised 13 December 2012; accepted 25 December 2012

\begin{abstract}
Mucus hypersecretion in airways is a common pathological change observed in chronic inflammatory diseases and asthma. We investigated the new role of cell attachment to the extracellular matrix (ECM) on the production of the airway mucus protein, MUC5AC mucin, in human airway epithelial cells, NCI-H292. MUC5AC levels of cells cultured on low adhesion plates were 10-fold higher than those of cells cultured on adhesion plates. Cells cultured on bovine serum albumin (BSA) coated plates, which produce low adhesion conditions, also induced the up-regulation of MUC5AC. Mucin staining by PAS and MUC5AC immunodetection confirmed that mucin proteins were overproduced under low adhesion conditions. The major adhesion molecule between cells and the ECM was integrins. A time-course experiment showed that the expression patterns of integrin $\beta 1$ and MUC5AC protein were inversely proportional. The inhibition of integrin $\beta 1$ induced an increase in MUC5AC production in cells cultured under adhesion conditions, but not under low adhesion conditions. These results suggested that cell attachment regulates MUC5AC production, which is up-regulated by low adhesion to the ECM, and MUC5AC production is inversely proportional to the function of integrin $\beta 1$.
\end{abstract}

Keywords: Asthma; Mucin; MUC5AC; Mucus; Integrin

\section{INTRODUCTION}

Asthma, a chronic inflammatory disease of the bronchial tubes, is characterized by hyperreactivity to various external stimuli, airway inflammations, and airway obstructions [1]. Airway obstructions are caused by bronchospasms and mucus build ups from airway epithelial cells. The mucus layer, which comprise water, ions, and

${ }^{*}$ Corresponding author. mucin glycoproteins, coats the airway epithelium and is essential for host defense against foreign pathogens or external irritants. Mucins, which are high-molecularweight and heavily-glycosylated proteins, are needed to maintain the the viscosity of the mucus layer and are secreted by the mucosal goblet cells [2]. Mucin proteins in the mucus layer are overproduced in the respiratory tracts of patients with asthma. Thereafter, the overproduction of the mucus layer by mucins causes airway obstruction $[3,4]$.

To date, over 20 types of mucin (MUC) genes have been identified. Eleven MUC genes (MUC1, 2, 3, 4, $5 \mathrm{AC}, 5 \mathrm{~B}, 6,7,8,13$, and 19) have been reported to be expressed in the lungs [5]. The mucus layer in the human airway epithelia comprise two major types of mucins, MUC5B and MUC5AC [4]. MUC5B and MUC5AC are involved in the pathogenesis of various respiratory infectious diseases, such as chronic obstructive pulmonary disease and they contribute to the amplification of inflammation [6-8].

MUC5B is the basic gel-forming mucin that is located in the airways of humans and mice, and MUC5AC is the principal gel-forming mucin that is overproduced in airway inflammation. MUC5AC mucin was initially identified in the lung mucus of a patient with asthma [9-12]. The mRNA levels of MUC5AC are moderately increased in the airway mucus of patients with asthma [13]. Furthermore, the levels of expression of a MUC5AC homo$\log$ are increased in murine models of asthma [14]. Thus, the production of MUC5AC is a signature of human asthma and murine models of asthma.

Many studies have described the regulation of MUC$5 \mathrm{AC}$ expression in airway epithelial cells as a target for asthma treatment. Bacterial inflammation in the airway induces morphological and proliferate changes in goblet cells and mucus hypersecretion. The main inducers of bacterial inflammation are lipopolysaccharides (LPSs) $[15,16]$. LPSs have been shown to upregulate the levels of expression of MUC5AC mRNA (by 2.4 times) in 
HT-29 goblet cells in vitro. Some Th2 cytokines, such as IL-4 and IL-13, are involved in the regulation of MUC$5 \mathrm{AC}$ production through the differentiation of epithelial cells into goblet cells, which produce the mucus layer, in animal models and humans in vivo [17,18]. The resulting remodeling of the epithelium and the associated mucus hypersecretion contribute to the overall morbidity and mortality of patients with asthma $[10,19,20]$. IL-4, IL-13, and TNF-alpha cytokines have been shown to increase epithelial cell mucus in animal models and human airway cells in vitro [21-24]. Various stimulations increase MUC5AC levels via EGFR-PI3K-Akt/ERK signaling pathway in human airway epithelial cells $[25,26]$.

The cell-cell adhesion molecule, E-cadherin, has been shown to be involved in the regulation of MUC5AC mucin production. E-cadherin promotes EGFR-mediated cell differentiation and MUC5AC production through a pathway that involves EGFR dephosphorylation [27]. In sparsely cultured NCI-H292 cells, E-cadherin inhibition activates EGFRs independent of Src and promotes MUC$5 \mathrm{AC}$ production through the ERK signaling pathway [28]. However, information on the involvement of cell attachment to the ECM is largely unknown.

The ECM provides not only mechanical support for cell structure, but also regulates in vivo cell differentiation and proliferation. The ECM is a complex of a number of molecules, including laminin, fibronectin, and collagen, and it adheres to cells through adhesion molecules. Integrins, which are major receptors that recognize and interact with the ECM, are heterodimers that are composed of $\alpha$ - and $\beta$-subunits. There are at least 16 $\alpha$-subunits and $8 \beta$-subunits on the cell surface in mammals. The signals from integrins are transregulated by talin, FAK, Src, and others to induce specific gene expression, but the relationship between the cell attachment to the ECM that involves the integrin signaling pathway and MUC5AC production is not completely understood [29].

Previously, it was reported that the ECM and an integrin/Src pathway might play an important role in the regulation of MUC5AC production in the NCI-H292 cell line. MUC5AC production is downregulated on type-IV collagen through a Src-independent pathway. In contrast, MUC5AC is upregulated on Matrigel or laminin through a Src-dependent pathway in NCI-H292 cells [30]. In previous studies, we found that the strength of the attachment of NCI-H292 cells to the ECM varies depending on the ECM components. These results showed a possibility that the strength of cell attachment to the ECM is involved in MUC5AC mucin production.

Our aim of this study was to evaluate the regulation of MUC5AC mucin production by cell attachment in the airway epithelial cells.

\section{MATERIALS AND METHODS}

\subsection{Cell Culture}

The human lung cancer cell line NCI-H292 was purchased from the American Type Culture Collection (MD, USA). NCI-H292 cells were cultured in RPMI-1640 (Sigma Aldrich, Tokyo, Japan) supplemented with 10\% fetal bovine serum (FBS, Cansera International, Etobicoke, Canada), 100 units $/ \mathrm{ml}$ penicillin (Gibco Oriental, Tokyo, Japan), and $100 \mu \mathrm{g} / \mathrm{ml}$ streptomycin (Gibco Oriental, Tokyo, Japan) in a $5 \% \mathrm{CO}_{2}$ incubator. The adherent cells were subcultured every 3 - 4 days by treatment with a trypsin-EDTA solution (Gibco Oriental, Tokyo, Japan). Cells were seeded on two types of culture plates: a 96-well plate (MS-8096F, Sumilon, Tokyo, Japan) as an adhesive plate, or a 96-well plate for a low-adhesive plate (MS8096R, Sumilon, Tokyo, Japan).

\subsection{Plate Coating with Fibronectin and BSA}

Polystyrene 96-well plates (MS-8096F or MS-8096R, Sumilon, Tokyo, Japan) were coated with $100 \mu \mathrm{l}$ of fibronectin (33 $\mu \mathrm{g} / \mathrm{ml}$, Asahi techno glass, Tokyo, Japan) in phosphate buffered saline (PBS: $0.01 \mathrm{M}$ phosphate buffer, $0.138 \mathrm{M} \mathrm{NaCl}, 0.0027 \mathrm{M} \mathrm{KCl}, \mathrm{pH} 7.4$ ) for $10 \mathrm{~h}$ at $4^{\circ} \mathrm{C}$. The plate was coated with bovine serum albumin (BSA, $0.1 \mathrm{mg} / \mathrm{ml}$ or $1 \mathrm{mg} / \mathrm{ml}$, TRACE, New Zealand) in PBS for $10 \mathrm{~h}$ at $4{ }^{\circ} \mathrm{C}$. The plates were treated with $100 \mu \mathrm{l}$ of PBS as controls.

\subsection{MUC5AC Protein Assay}

NCI-H292 cells were washed once with culture medium and suspended in the medium using a syringe with a $26 \mathrm{G}$ needle to obtain a single-cell suspension. Diluted cells (1 $\times 10^{4}$ cells in $100 \mu \mathrm{l}$ ) were added to the wells of coated plates and incubated for $3-30 \mathrm{~h}$ at $37^{\circ} \mathrm{C}$. After removal of the culture medium, the cells were harvested by lysis in Tris-buffered saline (TBS: $150 \mathrm{mM} \mathrm{NaCl}, 10 \mathrm{mM}$ Tris $\mathrm{pH} 7.5$ ) containing $0.1 \%$ SDS. A total of $40 \mu \mathrm{l}$ of the solution was blotted onto an Immobilon membrane (Millipore, MA, USA) with a Dot Blot Hybridization Manifold (48 well, SCIE-PLAS, Cambridge, UK). When measuring the soluble MUC5AC level in culturing medium, removed culture medium was used as samples and applied to Immobilon membrane. The membrane was treated with $4 \%$ skim milk (Gibco Oriental, Tokyo, Japan) in $0.2 \%$ Tween 20 -TBS (TBS-T) for $12 \mathrm{~h}$ at $4^{\circ} \mathrm{C}$ and then was incubated with mouse anti-human MUC5AC antibody (MS145-P1, 1:2000 in 4\% skim milk, Thermo scientific, Kanagawa, Japan) or with rabbit anti-human MUC5AC polyclonal antibody (sc-20118, Santa Cruz Biotechnology, CA, USA) in integrin inhibition experiment for $1 \mathrm{~h}$. The membrane was washed five times for 5 min each with TBS-T and then incubated with rabbit 
anti-mouse $\operatorname{IgG}(\mathrm{H}+\mathrm{L})(1: 2000$ in $4 \%$ skim milk, NA931V, GE Healthcare, Buckinghamshire, UK) or goat anti-rabbit $\operatorname{IgG}(\mathrm{H}+\mathrm{L})$ conjugated with horseradish peroxidase (1:2000 in 4\% skim milk, W401B, Promega, WI, USA) for $1 \mathrm{~h}$. After washing the membrane five times, the enzyme reactions were detected with an ECL Western blotting detection reagent (GE Healthcare, Buckinghamshire, UK) and a LAS-4000 image analyzer (Fujifilm, Tokyo, Japan).

\subsection{Histological Periodic Acid-Schiff (PAS) Staining}

NCI-H292 cells $\left(3.1 \times 10^{4}\right.$ cells $\left./ \mathrm{cm}^{2}\right)$ were cultured in two types of culture plates: a 96-well plate (MS-8096F, Sumilon, Tokyo, Japan) as a adhesive plate, or a 96-well plate for low adhesion plate (MS8096R, Sumilon, Tokyo, Japan), which were pretreated with PBS, fibronectin or $\mathrm{BSA}$, for $30 \mathrm{~h}$. The culturing medium was removed from the plates, fixed with ethanol-acetic acid (3:1) for $1 \mathrm{~h}$, and then treated with $0.5 \%$ periodate for $5 \mathrm{~min}$ and Schiff reagent (Wako, Tokyo, Japan) and $0.6 \%$ sodium metabisulfite in $0.05 \mathrm{~N} \mathrm{HCl}$ by standard PAS staining methods. The PAS-stained plates were counter-stained with $0.04 \%$ Giemsa solution (Wako, Tokyo, Japan) for 5 min. After being washed with deionized $\mathrm{H}_{2} \mathrm{O}$, the slides were immediately viewed using a fluorescence microscope (BZ-9000, Keyence, Tokyo, Japan).

\subsection{Immunohistochemistry}

For immunofluorescence analyses, $3.1 \times 10^{4}$ cells $/ 200 \mu 1$ of cells were seeded in chamber slides (177445, LabTek $^{\mathrm{TM}}$ Nalge Nunc International, NY, USA) which were coated with fibronectin, BSA or PBS. After $30 \mathrm{~h}$ of culture, the culturing medium was removed and the cells were washed once with PBS. Then, cells were fixed with methanol for $10 \mathrm{~min}$ at $-20^{\circ} \mathrm{C}$, and were treated with acetone for $5 \mathrm{~min}$ at $-20^{\circ} \mathrm{C}$. The cells were blocked with $1 \%$ of BSA in PBS for $30 \mathrm{~min}$ at room temperature, and were washed three times with PBS. The fixed and rinsed cells were incubated with mouse monoclonal anti MUC5AC antibody (MS145-P1, Thermo SCIENTIFIC, Kanagawa, Japan) as primary antibodies (1:1500 dilution in PBS) for $1 \mathrm{~h}$ at room temperature. Then, cells were washed three times with PBS and were incubated with anti-mouse IgG-FITC (\#238, MBL, Nagoya, Japan) as secondary antibodies (1:1500 dilution in PBS) for $1 \mathrm{~h}$ at room temperature. After being washed in PBS, the slides were immediately viewed using a fluorescence microscope (BZ-9000, Keyence, Tokyo, Japan).

\subsection{Cell Proliferation Assay}

Cell proliferation was assessed using a Cell Counting
Kit-8 (Dojindo, Kumamoto, Japan). NCI-H292 cells $(1 \times$ $10^{4}$ cells in $0.1 \mathrm{ml}$ ) were cultured on a 96-well plates for $30 \mathrm{~h}$ at $37^{\circ} \mathrm{C}$. After adding a reagent of the kit $(0.01 \mathrm{ml})$ to each well, the plate was incubated for $2 \mathrm{~h}$ at $37^{\circ} \mathrm{C}$. The cell growth was assessed by measuring the absorbance at $450 \mathrm{~nm}$ using a Model 550 microplate reader (BioRad, Tokyo, Japan).

\subsection{Cell Adhesion Assay}

A 96-well plate was treated with fibronectin, BSA or PBS as described above. A single-cell suspension $(1 \times$ $10^{4}$ cells) in $100 \mu$ RPMI supplemented with $0.1 \%$ BSA was added to each well. Cells were allowed to adhere for $120 \mathrm{~min}$ at $37^{\circ} \mathrm{C}$. The cells were subsequently washed with PBS to remove nonadherent cells. The adhered cells were stained with $50 \mu 1$ of $0.5 \%$ crystal violet (Sigma, Tokyo, Japan) in $20 \%$ methanol for $30 \mathrm{~min}$. The cells were washed with deionized water and were lysed in 50 $\mu 1$ of $30 \%$ acetic acid. Cell adhesion was calculated by measuring the absorbance of the eluted dye at $570 \mathrm{~nm}$ using a Model 550 microplate reader (BioRad, Tokyo, Japan).

\subsection{Immunoblot; Detection of Integrin $\beta 1$ and $\beta$-Actin}

The cells cultured on the ECM-coated plate were lysed in a conventional SDS sample buffer $(62.5 \mathrm{mM}$ Tris, $10 \%$ Glycerol, 2\% SDS, 0.01\% bromphenol blue, pH 6.8). The cell proteins were electrophoresed on SDS-PAGE with a CM-1005 gel apparatus (Cima Biotech, Tokyo, Japan), and then blotted onto a nitrocellulose membrane (Hybond ECL, GE Healthcare, Buckinghamshire, UK) with a M3001 transfer apparatus (Cima Biotech, Tokyo, Japan). The membrane was treated with $4 \%$ skim milk (WAKO, Tokyo, Japan) in TBS-T for $12 \mathrm{~h}$ at $4^{\circ} \mathrm{C}$ and then was incubated with a rabbit anti-integrin $\beta 1$ polyclonal antibody (4706S; Cell Signaling Technology, MA, USA) at 1:2000 dilution in Canget signal immunoreaction enhancer solution (NKB101, TOYOBO, Tokyo, Japan) for $1 \mathrm{~h}$. The membrane was washed five times for 5 min each with TBS-T and then incubated with anti-rabbit IgG antibody conjugated with horseradish peroxidase (W4011, Promega, WI, USA) at 1:2000 dilution for $1 \mathrm{~h}$. After washing the membrane five times, the enzyme reaction was detected with a Luminata Forte western HRP substrate (WBLUF0500, Millipore, MA, USA) and a LAS-4000 image analyzer (Fujifilm, Tokyo, Japan). Cellular $\beta$-actin was detected as a control using a rabbit anti- $\beta$-actin antibody (A5316, Sigma, MO, USA) at 1:2000 dilution and a rabbit anti-mouse $\operatorname{IgG}(\mathrm{H}+\mathrm{L})$ $(1: 2000$ in $4 \%$ skim milk, NA931V, GE healthcare, Buckinghamshire, UK). Then, blot membrane was incubated with Restore western blot Stripping buffer (21059, 
Thermo Scientific, IL, USA) for $15 \mathrm{~min}$ at room temperature, with shaking. The membrane was washed five times for 5 min each with TBS-T and then treated with 4\% skim milk (Gibco Oriental, Tokyo, Japan) in TBS-T for $12 \mathrm{~h}$ at $4^{\circ} \mathrm{C}$ for reblocking.

\subsection{Inhibition of Integrin Activity}

NCI-H292 cells $\left(1 \times 10^{4}\right.$ in $\left.0.1 \mathrm{ml}\right)$ were cultured on plates coated with PBS, fibronectin or BSA. The cells were treated with normal mouse IgG (Santa Cruz Biotechnology, CA, USA) and mouse anti-human integrin $\beta 1$-antibody (MAB1965, Millipore, MA, USA) at a final concentration of $50 \mu \mathrm{g} / \mathrm{ml}$ for $30 \mathrm{~h}$ at $37^{\circ} \mathrm{C}$. The level of MUC5AC proteins were detected by a method similar to that described in the MUC5AC protein assay. Antibodies of anti-human MUC5AC (1:2000, rabbit, sc-20118, Santa Cruz Biotechnology, CA, USA) and anti-rabbit IgG $(\mathrm{H}+$ L) conjugated with horseradish peroxidase (1:2000, W4011, Promega, WI, USA) were used in this experiment.

\subsection{Statistics}

Student's $t$-test. ${ }^{*} \mathrm{P}<0.05,{ }^{* *} \mathrm{P}<0.01$ compared with controls.

\section{RESULTS}

\subsection{A Reduction in Cell Attachment Induced an Up-Regulation of the Levels of MUC5AC Mucin}

We previously reported that culturing cells on BSAtreated plates induced an increase in MUC5AC production (by 2.5 times) [30]. BSA coating inhibits initial cell attachment to the carrier surface, and is a common method used to reduce cell attachment. This result suggested that cell attachment was a part of the regulation of MUC5AC production and that culturing cells under low adhesion conditions induced an up-regulation in the levels of MUC5AC mucin. To investigate the relationship between cell attachment and MUC5AC production, we evaluated the effects of cell attachment on MUC5AC production. We used low adhesion cell culturing plates, which were not treated for cell attachment, to culture the cells under low adhesion conditions and compared these with cells that had been cultured on treated adhesion plates for culturing cells under adhesion conditions. The cells were cultured for $30 \mathrm{~h}$ under both conditions. When the cells were cultured under low adhesion conditions, cell attachment was markedly reduced compared to the attachment of the cells that were cultured under adhesion conditions (Figure 1). In addition, the BSA-coated plates showed decreased cell attachment (Figure 1). The treatment with fibronectin recovered the adhesion of the cells

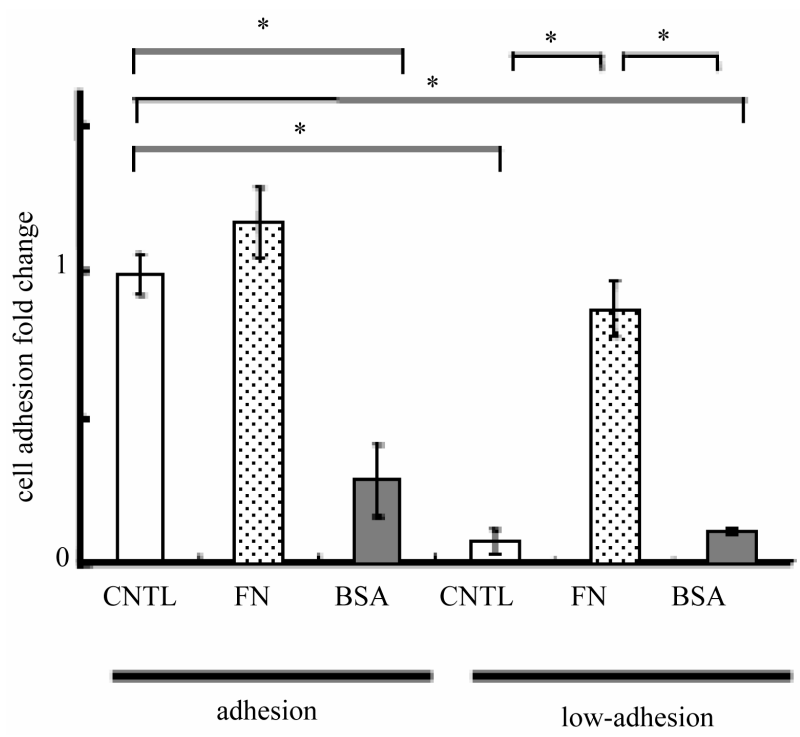

Figure 1. Cell attachment was reduced on BSA coated or on low adhesion plates. NCI-H292 cells were cultured in a 96-well plate, which is pretreated with PBS (CNTL), fibronectin (FN), and BSA (BSA) for $10 \mathrm{~h}$. Low adhesion plates were used for culturing cells under low adhesion condition. The adhered cells were stained with $0.5 \%$ Crystal violet and were calculated by measuring the absorbance of eluted dye at $570 \mathrm{~nm}$ with a Microplate reader. The fold changes are based on a control, which is treated with PBS in adhesion plates. The mean number of cells ( \pm s.d., $\mathrm{n}=5$, two-tailed Student's $t$-test) is shown. ${ }^{*} \mathrm{P}<$ 0.05 .

in low adhesion plates (Figure 1). The production of MUC5AC protein in cells that were cultured under both conditions was measured by immunoblot analysis. MUC5AC levels of cells cultured on low adhesion plates were 10-fold higher than those of cells cultured on adhesion plates (Figure 2(a)). The MUC5AC levels in cells, which were cultured on BSA coated adhesion plates, were increased dose-dependently (Figure 2(b)). The cells cultured on BSA coated low adhesion plates were also induced 10-fold increase of MUC5AC production. In the low adhesion plates coated with fibronectin, MUC5AC levels were not increased (Figure 3). Interestingly, the levels of soluble MUC5AC in the culturing medium were also increased under low adhesion conditions, or in the BSA treated plates, but the increase was not of the same degree as that observed in the cells (Figure 3).

\subsection{PAS Staining and Immunostaining of MUC5AC under Low Adhesion Conditions}

The periodic acid-schiff (PAS) stain stains glycoproteins, such as mucin protein, in mucus producing cells. In low adhesion conditions, mucin proteins were detected by PAS staining. Cells that were cultured on PBS, fibronectin or BSA treated adhesion plates or low adhesion 


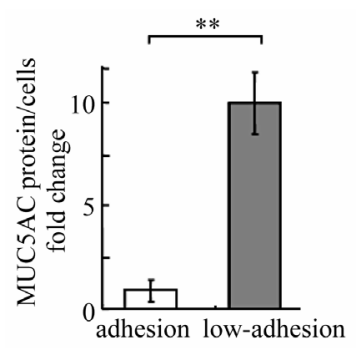

(a)

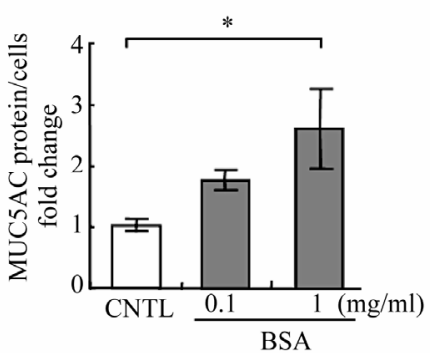

(b)
Figure 2. The level of MUC5AC production was up-regulated under low adhesion condition. (a) NCI-H292 cells were cultured in 96-well plates, which were pretreated with PBS in adhesion or low adhesion plates. Cells were sampled for $30 \mathrm{~h}$. The level of MUC5AC protein was detected by MUC5AC protein assay. The fold changes were based on controls which were treated with PBS in adhesion plates; (b) NCI-H292 cells were cultured in adhesion plates, which were precoated with PBS or $0.1 \mathrm{mg} / \mathrm{ml}$ or $1 \mathrm{mg} / \mathrm{ml}$ of BSA. Cells were sampled for $30 \mathrm{~h}$. The level of MUC5AC protein was detected by MUC5AC protein assay. The fold increases were based on controls which were treated with PBS. The mean number of cells $( \pm$ s.d., $n=5$, two-tailed Student's $t$-test) is shown. ${ }^{*} \mathrm{P}<0.05,{ }^{* *} \mathrm{P}<0.01$. The fold changes were normalized for cell number. Representative results from four independent experiments were shown.

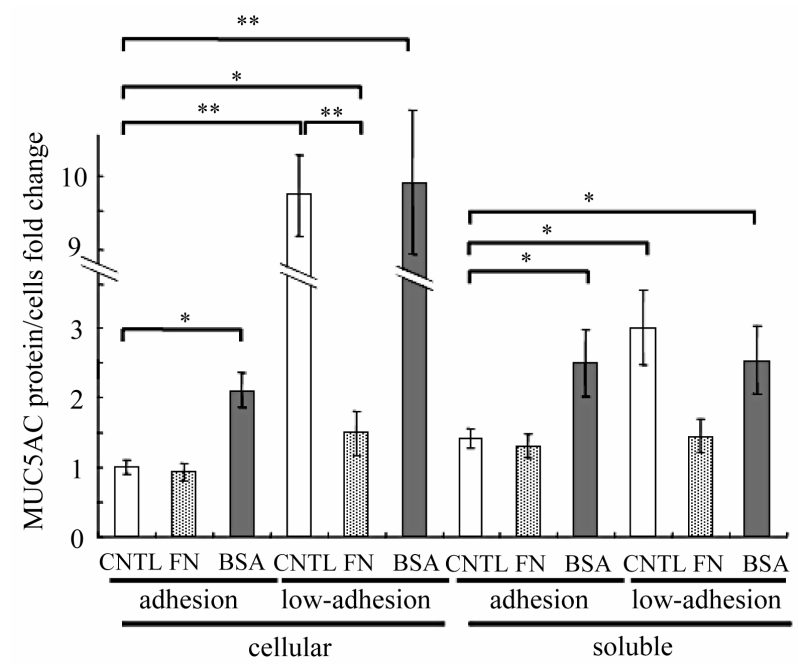

Figure 3. Cellular and soluble MUC5AC level were up-regulated under low adhesion conditions. NCI-H292 cells were cultured in a 96-well plate under an adhesion or low-adhesion condition, which were pretreated with PBS, fibronectin, or BSA. Cellular and soluble proteins, which were soluble in culturing medium, were sampled for $30 \mathrm{~h}$. The level of MUC5AC protein was detected by MUC5AC protein assay. The fold changes were based on controls which were treated with PBS under adhesion condition. The mean number of cells $( \pm$ s.d., $\mathrm{n}=$ 5 , two-tailed Student's $t$-test) is shown. ${ }^{*} \mathrm{P}<0.05,{ }^{* *} \mathrm{P}<0.01$. The fold changes were normalized for cell number. Representative results from three independent experiments were shown.

plates were stained with PAS and Giemsa. The number of PAS-positive cells was significantly increased on BSA-coated or low adhesion plates compared to that on
PBS or fibronectin coated plates (Figures 4(a)-(f)). These results confirmed the results that a reduction of cell attachment induced an increase in mucin production.

Next, the production of MUC5AC protein was examined by immunostaining with specific antibodies. The number of MUC5AC-positive cells was increased on BSA-coated plates compared to that in control cells (Figures 4(g)-(i)).

These staining results confirmed that MUC5AC mucin production was increased under low adhesion conditions.

\subsection{MUC5AC Production Was Inversely Proportional to the Level of Integrin $\beta 1$}

Our results suggested that cell attachment suppressed MUC5AC production. The main molecules involved in cell-ECM adhesion are the integrins. Hence, in the next experiment, we investigated the function of integrins in the regulation of MUC5AC production under low adhesion conditions.

In a previous report, we found that the inhibition of the (a)

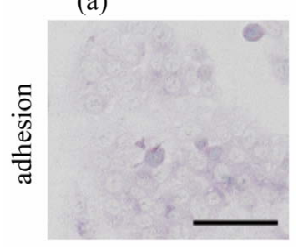

(d)

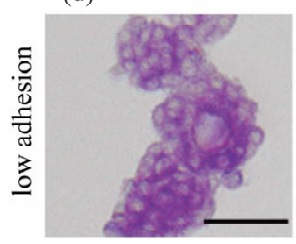

(g)

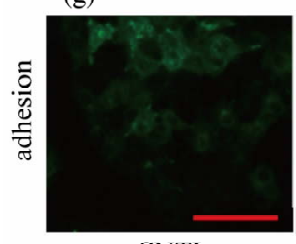

CNTL (b)

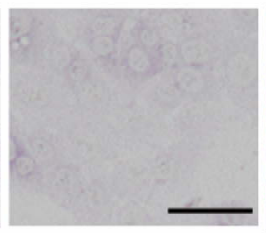

(e)

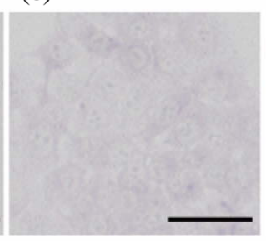

(h)

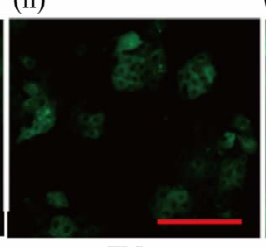

FN (c)

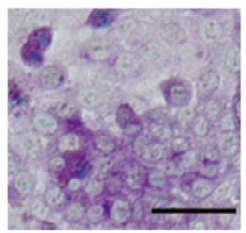

(1)

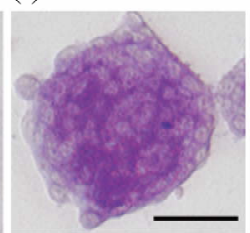

(i)

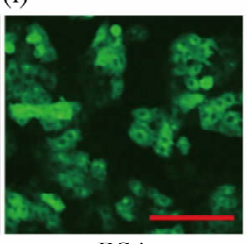

BSA
Figure 4. Mucus and MUC5AC production was up-regulated under low adhesion condition. NCI-H292 cells $\left(3.1 \times 10^{4}\right.$ cells $/ \mathrm{cm}^{2}$ ) were cultured on adhesive plates (a)-(c), (g)-(i) or on low adhesion plates (d), (e), and (f) for $30 \mathrm{~h}$. The plates were pretreated with PBS (a), (d), (g), fibronectin (b), (e), (h), and BSA (c), (f), (i) respectively. Cells were stained with PAS and Wright-Giemsa (a)-(f). MUC5AC protein was detected in cells by immuno-histochemical method (g)-(i). NCI-H292 cells $(1 \times$ $10^{4}$ cells/well) were cultured for $30 \mathrm{~h}$ on a Lab-Tek Chamber Slide, and were pre-coated with fibronectin (FN) or BSA (g)(i). MUC5AC protein was detected with specific antibodies. The magnification was $\times 100$, and black or red bars indicate 200 $\mu \mathrm{m}$. 
integrin $\beta 1$ subunit induced an up-regulation of MUC$5 \mathrm{AC}$ production. These results suggested that the cell attachment that was induced by integrin $\beta 1$ was involved in the regulation of MUC5AC production in cells. We measured the time course of the changes in the expression level of integrin $\beta 1$ and MUC5AC protein in cells. Integrin $\beta 1$ on the cells was digested by trypsinEDTA treatment, and the treated cells were seeded on normal adhesion culture plates. An immunoblot experiment showed that integrin $\beta 1$ was gradually synthesized and detected $24 \mathrm{~h}$ after seeding (Figure 5). In contrast, the levels of expression of MUC5AC were decreased markedly $24 \mathrm{~h}$ after seeding and they remained low until $30 \mathrm{~h}$ (Figure 5).

Next, we measured the levels of expression of integrin $\beta 1$ in cells that were cultured on fibronectin-coated, BSA-coated adhesion or low adhesion plates for $30 \mathrm{~h}$. We found that the levels of expression of integrin $\beta 1$ protein were decreased in fibronectin-coated low adhesion plates, but other samples were not showed obvious change under adhesion or low adhesion conditions (Figure 6(a)). Next, we investigated whether integrin $\beta 1$ was involved in the up-regulation of MUC5AC production under low adhesion conditions. Cells were cultured with inhibitory antibodies of integrin $\beta 1$ or with control IgG on fibronectin coated or BSA coated adhesion plates or low adhesion plates for $30 \mathrm{~h}$. The inhibition of integrin

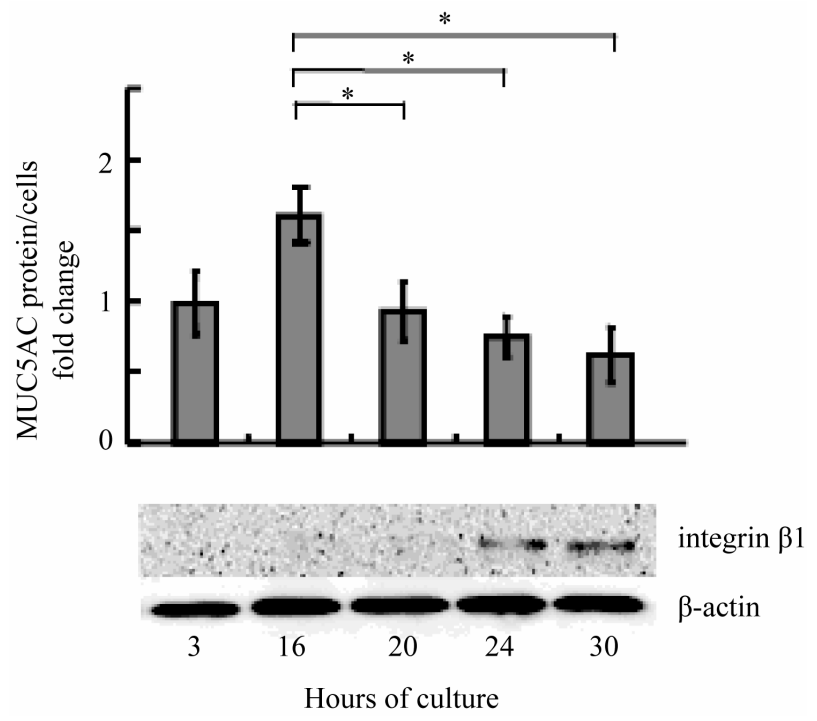

Figure 5. MUC5AC production was inversely proportional to the expression level of integrin $\beta 1$. NCI-H292 cells were cultured in a 96-well plate. Cells were sampled at the times indicated. The amount of integrin $\beta 1$ and $\beta$-actin was detected with specific antibody, respectively. The amount of MUC5AC protein was detected according to the MUC5AC protein assay. The fold changes were based on the sample at $3 \mathrm{~h}$ of culture $( \pm$ s.d., $\mathrm{n}=5$, two-tailed Student's $t$-test) is shown. ${ }^{*} \mathrm{P}<0.05$. The fold changes were normalized for cell number. Representative results from three independent experiments were shown.

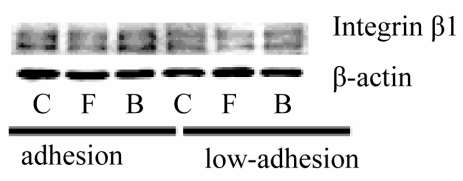

(a)

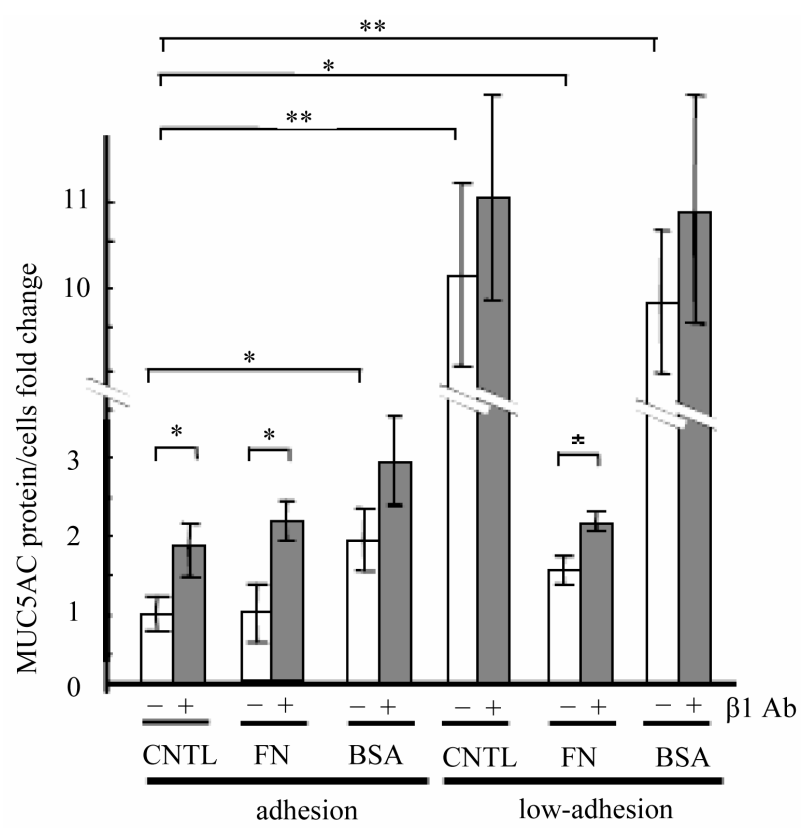

(b)

Figure 6. Inhibition of integrin $\beta 1$ up-regulated MUC5AC productions under adhesion condition. (A) NCI-H292 cells $(1 \times$ $10^{4}$ cells/well) were cultured in a 96-well plate, which was precoated with PBS (C), fibronectin (F) or BSA (B) on an adhesion plates or on low adhesion plates. Cells were sampled for $30 \mathrm{~h}$, and integrin $\beta 1$ and $\beta$-actin protein was detected by immunoblot with specific antibodies. (B) NCI-H292 cells were cultured in 96-well plates, which were precoated with PBS (CNTL), fibronectin (FN) or BSA (BSA) with control mouse IgG (-) or anti integrin $\beta 1$ inhibitory antibody $(+)$. Low adhesion plates were used for low adhesion condition. After $30 \mathrm{~h}$ of culture, the levels of cellular MUC5AC proteins were detected. The fold increases were based on a control, which was treated with PBS under an adhesion condition. The mean number of cells ( \pm s.d., $\mathrm{n}=5$, two-tailed Student's $t$-test) is shown. ${ }^{*} \mathrm{P}<$ $0.05,{ }^{* *} \mathrm{P}<0.01$. The fold changes were normalized for cell number. Representative results from three independent experiments were shown.

$\beta 1$, which was induced with specific antibodies, resulted in an up-regulation of MUC5AC mucin under adhesion conditions, but not under low adhesion conditions. These results suggested that the function of integrin $\beta 1$ under low adhesion conditions is already decreased compared to that in the control cells. When cells were cultured on fibronectin-coated adhesion plates, MUC5AC production and the amount of increase of MUC5AC that was induced by integrin $\beta 1$ inhibition were equivalent to PBS treated control (Figure 6(b)).

These results, which suggested that both the functional 
level and the expression level of integrin $\beta 1$ were inversely proportional to MUC5AC production, supported our hypothesis that the activity of integrin $\beta 1$ strengthens the cell attachment and suppresses the production of MUC5AC in NCI-H292 cells.

\section{DISCUSSION}

Airway mucus obstructions have long been recognized as a principal cause of death in patients with asthma. The markedly up-regulated production of MUC5AC mucin that occurs with the stimulated hypersecretion of mucus leads to airflow obstructions in asthma patients. However, the molecular mechanisms of mucus overproduction and secretion have not been understood until recently. Now, many studies have been conducted on the regulation of mucin production in airway epithelia. It has been reported that MUC5AC production is regulated by Th2like cytokines, bacterial products, and cell-cell adhesion, but the effects of cell-ECM adhesion to mucus expression have been poorly reported. We previously found that the signal from the ECM to cells is strongly involved in the regulation of MUC5AC mucin production and that the strength of cell attachment to the ECM varies relative to the ECM components [30]. In this report, we proposed that cell attachment might suppress MUC5AC production.

It has been reported that asthma is associated with extensive remodeling of the composition of the ECM. The basement membrane of the surface epithelium of the airway contains a number of ECM components, such as collagen I, collagen III, fibronectin, or laminin. These components were produced from activated myofibroblasts and accumulated. In asthma patients, thickening of the basement membrane is observed, and the composition of these ECM components are massively altered [31-33]. Asthma model mice, which are sensitized by ovalbumin, show increased levels of the expression of the laminin-1 isoform and the laminin-1 receptor in airways compared to controls [34]. The asthmatic smooth muscle (ASM) cells in airway epithelia show progressive proliferation and alterations in their ECM profiles in asthma patients. Increases in of ASM mass and changes in ECM production are observed in patients with asthma [35,36].

The remodeling and turnover of ECM components are highly regulated by metalloproteinases (MMPs) and their inhibitor, the tissue inhibitor of metalloproteinases (TIMP). MMPs, which are a group of enzymes that are capable of degrading the ECM, are secreted by stromal or inflammatory cells [37]. The levels of expression of MMP-9, which is a family gene of MMPs, or TIMP-1 are obviously increased in patients with mild asthma [38]. In addition, MMP-9 production, which is increased in children with asthma, correlates with lung function [39].
These reports, together with our results, suggest that the remodeling of ECM components should induce changes in cell attachment, which induce mucin overproduction in vivo in asthma patients. More investigations on the changes of the composition of the ECM components and of MMP activity in vivo and in vitro are needed.

Our results showed that culturing cells under low adhesion conditions induced MUC5AC overproduction (Figures 1-3). The immunodetection of mucin or MUC$5 \mathrm{AC}$ confirmed this increase (Figure 4). This increase of MUC5AC is almost cellular change (Figure 3). These results suggested that the activation of MUC5AC production is, at least partially, dependent on the strength of cell adhesion to the ECM and that cell attachment inhibits mucin overproduction.

The integrin subunit, which is the major molecule that is involved in cell-cell and cell-ECM adhesive interactions, transduces signals from the ECM to the cells. Integrins constitute a family of transmembrane receptors that are composed of heterodimeric complexes of linked $\alpha$ and $\beta$ subunits. The $\beta$ subunits are important in signal transduction from the ECM to cells, and the working integrin- $\beta$ subunit differs according to the components of the ECM [29]. Among the integrin family genes, the integrin $\beta 1$ subunit is the most common subunit that is involved in the attachment to representative ECM components, such as fibronectin, laminin, and collagen. As shown in our previous report, the inhibition of integrin$\beta 1$ function induced an up-regulation of MUC5AC production. Our time-course experiment showed that the levels of expression of integrin $\beta 1$ were inversely proportional to MUC5AC production in cells (Figure 5). From our results, cell attachment was largely decreased when cells were cultured on BSA-coated or low adhesion plates compared to those cultured on adhesion plates. However, the amount of integrin $\beta 1$ that was expressed on the cells remained unchanged (Figure 6(a)). These results suggested that the function of integrin $\beta 1$ was largely inhibited under low adhesion circumstances and it induced the up-regulation of MUC5AC mucin production. In our results, the inhibition of integrin $\beta 1 \mathrm{did}$ not induce an increase of MUC5AC production under low adhesion conditions (Figure 6(b)). These results suggested that integrin $\beta 1$ was less effective under low adhesion conditions. In addition, we found that the levels of expression of integrin $\beta 1$ protein were decreased in fibronectin coated low adhesion plates (Figure 6(a)). This result may explain the small increase of MUC5AC levels in fibronectin coated low adhesion plates (Figures 3 and 6(b)). These results also suggested that the activity of integrin $\beta 1$ down-regulated MUC5AC production. However, it should be noted that there is the possibility that another $\beta$ subunit of integrin might be involved in MUC5AC production. Therefore, we would like to in- 
vestigate the function of other $\beta$ subunits, like $\beta 3$ and $\beta 5$.

Our results suggested that the regulation of cell attachment inhibits MUC5AC production through integrin $\beta 1$ function, and that the effects of ECM on the regulation of MUC5AC production are induced by the strength of cell attachment. The strength of cell attachment and the level of MUC5AC production are affected markedly by ECM components [30]. Therefore, regulating the expression of ECM components, such as collagen IV or laminin, might be used for asthma medications. Because cell attachment involved integrin $\beta 1$ function, the control of integrin $\beta 1$ and its downstream signaling pathway might be a more useful medication for asthma than the control of mucin production in airway epithelia.

In the future, we plan to investigate as to which pathways are activated by different ECM components in a time-dependent manner. The pathways that act through ERK and NF- $\mathrm{KB}$-based transcriptional mechanisms are involved in MUC5AC regulation [40]. ERK and NF-kB pathways are activated in asthma patients and in the lower stream of the integrin-activating pathway. With transgenic mice, the activation of NF- $\mathrm{kB}$ in the airway epithelium has been shown to be necessary to induce airway inflammation and mucus hypersecretion $[41,42]$. Additional studies on the effects of the signal transduction pathways involving integrin signaling and the ERKsignaling pathway on various ECM components in patients with asthma will be useful in the specific inhibition of MUC5AC mucin overproduction and in the treatment of asthma.

\section{CONCLUSION}

We have investigated MUC5AC production under low adhesion culturing conditions in human airway epithelial cells and we have demonstrated that low cell adhesion condition induced a marked up-regulation of MUC5AC production. We found that the amount of integrin $\beta 1$ was inversely proportional to the level of MUC5AC and the inhibition of integrin $\beta 1$ function induced an up-regulation of MUC5AC production in NCI-H292 cells. These findings suggest that cell attachment involving integrin $\beta 1$ function down-regulates MUC5AC production.

\section{ACKNOWLEDGEMENTS}

This work was supported in part by a Grant-in-Aid for Scientific Research_(C) from JSPS (No. 23570238) and Akita Prefectural University President's Research Project.

\section{REFERENCES}

[1] Knowles, M.R. and Boucher, R.C. (2002) Mucus clearance as a primary innate defense mechanism for mammalian airways. The Journal of Clinical Investigation,
109, 571-577.

[2] Rose, M.C., Nickola, T.J. and Voynow, J.A. (2001) Airway mucus obstruction: Mucin glycoproteins, MUC gene regulation and goblet cell hyperplasia. American Journal of Respiratory Cell and Molecular Biology, 25, 533-537.

[3] Kaliner, M., Shelhamer, J.H., Borson, B., Nadel, J., Patow, C. and Marom, Z. (1986) Human respiratory mucus. The American Review of Respiratory Disease, 134, 612621.

[4] Rose, M.C. and Voynow, J.A. (2006) Respiratory tract mucin genes and mucin glycoproteins in health and disease. Physiological Reviews, 86, 245-278. doi: 10.1152/physrev.00010.2005

[5] Thai, P., Loukoianov, A., Wachi, S. and Wu, R. (2008) Regulation of airway mucin gene expression. Annual Review of Physiology, 70, 405-429. doi:10.1146/annurev.physiol.70.113006.100441

[6] Li, J.D. (2003) Exploitation of host epithelial signaling networks by respiratory bacterial pathogens. Journal of Pharmacological Sciences, 91, 1-7. doi:10.1254/jphs.91.1

[7] Fahy, J.V. and Dickey, B.F. (2010) Airway mucus function and dysfunction. The New England Journal of Medicine, 363, 2233-2247. doi:10.1056/NEJMra0910061

[8] Vestbo, J., Lange, P. and Hansen, E.F. (2002) Chronic obstructive pulmonary disease. World COPD Day 2002. Ugeskrift for Laeger, 164, 5510.

[9] Rose, M.C., Kaufman, B. and Martin, B.M. (1989) Proteolytic fragmentation and peptide mapping of human carboxyamidomethylated tracheobronchial mucin. The Journal of Biological Chemistry, 264, 8193-8199.

[10] Fahy, J.V. (2002) Goblet cell and mucin gene abnormalities in asthma. Chest, 122, 320S-326S.

[11] Thornton, D.J., Carlstedt, I., Howard, M., Devine, P.L., Price, M.R. and Sheehan, J.K. (1996) Respiratory mucins: Identification of core proteins and glycoforms. The Biochemical Journal, 316, 967-975.

[12] Hoessli, D.C., Davidson, E.A., Schwarz, R.T. and Nasirud, D. (1996) Glycobiology of Plasmodium falciparum: An emerging area of research. Glycoconjugate Journal, 13, 1-3. doi:10.1007/BF01049673

[13] Ordonez, C.L., Khashayar, R., Wong, H.H., Ferrando, R., Wu, R., Hyde, D.M., Hotchkiss, J.A., Zhang, Y., Novikov, A., Dolganov, G. and Fahy, J.V. (2001) Mild and moderate asthma is associated with airway goblet cell hyperplasia and abnormalities in mucin gene expression. American Journal of Respiratory and Critical Care Medicine, 163, 517-523.

[14] Zuhdi, A.M., Piazza, F.M., Selby, D.M., Letwin, N., Huang, L. and Rose, M.C. (2000) Muc-5/5ac mucin messenger RNA and protein expression is a marker of goblet cell metaplasia in murine airways. American Journal of Respiratory Cell and Molecular Biology, 22, 253-260.

[15] Smirnova, M.G., Guo, L., Birchall, J.P. and Pearson, J.P. (2003) LPS up-regulates mucin and cytokine mRNA expression and stimulates mucin and cytokine secretion in goblet cells. Cellular immunology, 221, 42-49. doi:10.1016/S0008-8749(03)00059-5 
[16] Mohamed, F.B., Garcia-Verdugo, I., Medina, M., Balloy, V., Chignard, M., Ramphal, R. and Touqui, L. (2012) A crucial role of flagellin in the induction of airway mucus production by Pseudomonas aeruginosa. PLoS One, 7, e39888. doi:10.1371/journal.pone.0039888

[17] Kondo, M., Tamaoki, J., Takeyama, K., Nakata, J. and Nagai, A. (2002) Interleukin-13 induces goblet cell differentiation in primary cell culture from Guinea pig tracheal epithelium. American Journal of Respiratory Cell and Molecular Biology, 27, 536-541.

[18] Kondo, M., Tamaoki, J., Takeyama, K., Isono, K., Kawatani, K., Izumo, T. and Nagai, A. (2006) Elimination of IL-13 reverses established goblet cell metaplasia into ciliated epithelia in airway epithelial cell culture. Allergology International, 55, 329-336. doi:10.2332/allergolint.55.329

[19] Morcillo, E.J. and Cortijo, J. (2006) Mucus and MUC in asthma. Current Opinion in Pulmonary Medicine, 12, 1-6. doi:10.1097/01.mcp.0000198064.27586.37

[20] Thornton, D.J., Rousseau, K. and McGuckin, M.A. (2008) Structure and function of the polymeric mucins in airways mucus. Annual Review of Physiology, 70, 459-486. doi:10.1146/annurev.physiol.70.113006.100702

[21] Iwashita, J., Sato, Y., Sugaya, H., Takahashi, N., Sasaki, H., Abe, T. (2003) mRNA of MUC2 is stimulated by IL-4, IL-13 or TNF-alpha through a mitogen-activated protein kinase pathway in human colon cancer cells. Immunology and Cell Biology, 81, 275-282. doi:10.1046/j.1440-1711.2003.t01-1-01163.x

[22] Zhu, Z., Homer, R.J., Wang, Z., Chen, Q., Geba, G.P., Wang, J., Zhang, Y. and Elias, J.A. (1999) Pulmonary expression of interleukin-13 causes inflammation, mucus hypersecretion, subepithelial fibrosis, physiologic abnormalities, and eotaxin production. The Journal of Clinical Investigation, 103, 779-788. doi:10.1172/JCI5909

[23] Fujisawa, T., Ide, K., Holtzman, M.J., Suda, T., Suzuki, K., Kuroishi, S., Chida, K. and Nakamura, H. (2008) Involvement of the p38 MAPK pathway in IL-13-induced mucous cell metaplasia in mouse tracheal epithelial cells. Respirology, 13, 191-202. doi:10.1111/j.1440-1843.2008.01237.x

[24] Tanabe, T., Fujimoto, K., Yasuo, M., Tsushima, K., Yoshida, K., Ise, H. and Yamaya, M. (2008) Modulation of mucus production by interleukin-13 receptor alpha2 in the human airway epithelium. Clinical and Experimental Allergy, 38, 122-134. doi:10.1111/j.1365-2222.2007.02871.x

[25] Li, J.D., Feng, W., Gallup, M., Kim, J.H., Gum, J., Kim, Y. and Basbaum, C. (1998) Activation of NF-kappaB via a Src-dependent Ras-MAPK-pp90rsk pathway is required for Pseudomonas aeruginosa-induced mucin overproduction in epithelial cells. Proceedings of the National Academy of Sciences of the United States of America, 95, 5718-5723. doi:10.1073/pnas.95.10.5718

[26] Yang, J., Li, Q., Zhou, X.D., Kolosov, V.P. and Perelman, J.M. (2011) Naringenin attenuates mucous hypersecretion by modulating reactive oxygen species production and inhibiting NF-kappaB activity via EGFR-PI3K-Akt/ERK MAPKinase signaling in human airway epithelial cells.
Molecular and Cellular Biochemistry, 351, 29-40.

[27] Kim, S., Schein, A.J. and Nadel, J.A. (2005) E-cadherin promotes EGFR-mediated cell differentiation and MUC5AC mucin expression in cultured human airway epithelial cells. American Journal of Physiology-Lung Cellular and Molecular Physiology, 289, L1049-1060.

[28] Iwashita, J., Ose, K., Ito, H., Murata, J. and Abe, T. (2011) Inhibition of E-cadherin dependent cell-cell contact promotes MUC5AC mucin production through the activation of epidermal growth factor receptors. Bioscience, Biotechnology, and Biochemistry, 75, 688-693. doi: $10.1271 / \mathrm{bbb} .100830$

[29] Hynes, R.O. (2002) Integrins: Bidirectional, allosteric signaling machines. Cell, 110, 673-687. doi:10.1016/S0092-8674(02)00971-6

[30] Iwashita, J., Yamamoto, T., Sasaki, Y. and Abe, T. (2009) MUC5AC production is downregulated in NCI-H292 lung cancer cells cultured on type-IV collagen. Molecular and Cellular Biochemistry, 337, 65-75. doi:10.1007/s11010-009-0286-Z

[31] Roche, W.R., Beasley, R., Williams, J.H. and Holgate, S.T. (1989) Subepithelial fibrosis in the bronchi of asthmatics. Lancet, 1, 520-524. doi:10.1016/S0140-6736(89)90067-6

[32] Altraja, A., Laitinen, A., Virtanen, I., Kampe, M., Simonsson, B.G., Karlsson, S.E., Hakansson, L., Venge, P., Sillastu, H. and Laitinen, L.A. (1996) Expression of laminins in the airways in various types of asthmatic patients: A morphometric study. American Journal of Respiratory Cell and Molecular Biology, 15, 482-488.

[33] Chakir, J., Laviolette, M., Boutet, M., Laliberte, R., Dube, J. and Boulet, L.P. (1996) Lower airways remodeling in nonasthmatic subjects with allergic rhinitis. Laboratory Investigation, 75, 735-744.

[34] Christie, P.E., Jonas, M., Tsai, C.H., Chi, E.Y. and Henderson Jr., W.R. (2004) Increase in laminin expression in allergic airway remodelling and decrease by dexamethasone. The European Respiratory Journal, 24, 107-115. doi:10.1183/09031936.04.00013303

[35] Chung, K.F. (2000) Airway smooth muscle cells: Contributing to and regulating airway mucosal inflammation? The European Respiratory Journal, 15, 961-968. doi:10.1034/j.1399-3003.2000.15e26.x

[36] Johnson, P.R. (2001) Role of human airway smooth muscle in altered extracellular matrix production in asthma. Clinical and Experimental Pharmacology \& Physiology, 28, 233-236. doi:10.1046/j.1440-1681.2001.03426.x

[37] Massova, I., Kotra, L.P., Fridman, R. and Mobashery, S. (1998) Matrix metalloproteinases: Structures, evolution, and diversification. FASEB Journal, 12, 1075-1095.

[38] Mattos, W., Lim, S., Russell, R., Jatakanon, A., Chung, K.F. and Barnes, P.J. (2002) Matrix metalloproteinase-9 expression in asthma: Effect of asthma severity, allergen challenge, and inhaled corticosteroids. Chest, 122, 15431552. doi:10.1378/chest.122.5.1543

[39] Karakoc, G.B., Yukselen, A., Yilmaz, M., Altintas, D.U. and Kendirli, S.G. (2012) Exhaled breath condensate MMP-9 level and its relationship with asthma severity 
and interleukin-4/10 levels in children. Annals of Allergy, Asthma \& Immunology, 108, 300-304.

doi:10.1016/j.anai.2012.02.019

[40] Fujisawa, T., Velichko, S., Thai, P., Hung, L.Y., Huang, F. and $\mathrm{Wu}, \mathrm{R}$. (2009) Regulation of airway MUC5AC expression by IL-1beta and IL-17A: The NF-kappaB paradigm. Journal of Immunology, 183, 6236-6243. doi:10.4049/jimmunol.0900614

[41] Broide, D.H., Lawrence, T., Doherty, T., Cho, J.Y., Miller, M., McElwain, K., McElwain, S. and Karin, M.
(2005) Allergen-induced peribronchial fibrosis and mucus production mediated by IkappaB kinase beta-dependent genes in airway epithelium. Proceedings of the $\mathrm{Na}$ tional Academy of Sciences of the United States of America, 102, 17723-17728. doi:10.1073/pnas.0509235102

[42] Poynter, M.E., Cloots, R., van Woerkom, T., Butnor, K. J., Vacek, P., Taatjes, D.J., Irvin, C.G. and JanssenHeininger, Y.M. (2004) NF-kappa B activation in airways modulates allergic inflammation but not hyperresponsiveness. Journal of Immunology, 173, 7003-7009. 